\title{
Enhancement of Methane Conversion Using Electric Fields
}

\author{
Quarterly Report \\ January 1 - March 31, 1997
}

Work Performed Under Contract No.: DE-FG21-94MC31170

For

U.S. Department of Energy

Office of Fossil Energy

Federal Energy Technology Center

Morgantown Site

P.O. Box 880

Morgantown, West Virginia 26507-0880

By

Office of Research Administration

The University of Oklahoma

1000 Asp Avenue, Suite 314

Norman, Oklahoma 73019-0430 


\section{Disclaimer}

This report was prepared as an account of work sponsored by an agency of the United States Government. Neither the United States Government nor any agency thereof, nor any of their employees, makes any warranty, express or implied, or assumes any legal liability or responsibility for the accuracy, completeness, or usefulness of any information, apparatus, product, or process disclosed; or represents that its use would not infringe privately owned rights. Reference herein to any specific commercial product, process, or service by trade name, trademark, manufacturer, or otherwise does not necessarily constitute or imply its endorsement, recommendation, or favoring by the United States Government or any agency thereof. The views and opinions of authors expressed herein do not necessarily state or reflect those of the United States Government or any agency thereof. 


\section{DISCLAMIER}

Portions of this document may be illegible in electronic image products. Images are produced from the best avaliable original docoment. 


\section{PROJECT ABSTRACT}

The goal of this project is the development of novel, economical, processes for the conversion of natural gas to more valuable projects such as methanol, ethylene and other organic oxygenates or higher hydrocarbons. The methodologies of the project are to investigate and develop low temperature electric discharges and electric fieldenhanced catalysis for carrying out these conversions. In the case of low temperature discharges, the conversion is carried out at ambient temperature which in effect trades high temperature thermal energy for electric energy as the driving force for conversion. The low operating temperatures relax the thermodynamic constraints on the product distribution found at high temperature and also removes the requirements of large thermal masses required for current technologies. With the electric field-enhanced conversion, the operating temperatures are expected to be below those currently required for such processes as oxidative coupling, thereby allowing for a higher degree of catalytic selectivity while maintaining high activity. 


\section{TABLE OF CONTENTS}

ABSTRACT

ii

QUARTERLY OVERVIEW

1

APPENDIX (discharge/geometric phenomena discussion)

2 


\section{QUARTERLY OVERVIEW}

We are continuing experiments, however no significant new results were completed. We are still working on kinetics in the non-barrier discharge system. We completed repair of one GC and upgrading a second so that on the hydrocarbon only systems we can detect products up through the C5s and hydrogen production for all of the systems. We presented papers at the AIChE Spring Meeting and the DOE-Gas Contractors' Conference, both in Houston in March. Attached is a brief review of discharge

phenonmena with some discussion on effects of geometry and electrical parameters, an area that we are now attempting to further develop our understanding. 


\section{APPENDIX}

A DISCUSSION OF DICHARGE PHENOMENA AND FUTURE STUDIES ON GEOMETRICAL AND ELECTRICAL PARAMETERS 


\section{Discharge Phenomena in Methane Conversion}

\section{The Electric Discharge}

In its normal state, a gas is nearly a perfect insulator. In fact, high-pressure gases can be used to insulate high-voltage machines like X-ray generators.

However, if an electric potential of sufficient magnitude is applied across the gas between two metal electrodes, the gas can become a nearly perfect conductor. The gas gap is capable of passing high currents and then recovering its original insulating state almost immediately. The sudden transition of a gas from an insulator to a conductor is referred to as an electric breakdown.

The spectacular brilliance of many electric breakdowns occurring between two metal electrodes often begins at the quantum level. Normally, an electron at the surface of an electrode has a potential energy lower than that of an electron outside the metal by an amount $\chi$. The electrons in the metal have kinetic energies up to a maximum value $\zeta$ in accordance with Fermi-Dirac statistics. The potential barrier or work function $\phi$ of the metal is the work necessary to remove an electron from the. metal and is equal to the value $(\chi-\zeta)$. A strong electric field applied to the surface of an electrode can reduce or "thin" the potential barrier between the two electrodes and lead to field emission. Field emission is an extremely small ion current that occurs as electrons "leak" from the surface of the electrode into the gas gap. A wave mechanical approach to the problem shows that the electrons in the metal do 
not have to overcome the potential barrier but can tunnel through the barrier by virtue of their wave properties. This "leaking" of electrodes is known as the "tunnel effect."

Once the electrons escape into the gas gap, they immediately accelerate toward the anode. As the electrons travel through the gas gap, they collide with the neutral molecules of the gas species. The collisions between these neutral molecules and the electrons are usually elastic, that is, they bounce off one another with no change. If, however, an electron possesses a kinetic energy greater than that of the ionization energy of the neutral gas molecule, ionization can occur. Electrons from the gas molecule are stripped away and also begin to accelerate toward the anode, ionizing more molecules along their way. After a short period of time, a large quantity of electrons and positive and negative ions are present in the gas. The current between the electrodes quickly rises until a final flashover, or electric breakdown, occurs.

Ionization is not the only collision mechanism to take place within the gas gap. A summary of all the major collisions that can occur are shown in Table 1. These collisions are collectively known as electric discharge phenomenon. Many of the collisions may produce an active chemical species that can react to form a new species. The electric discharge process can be used in this manner as a tool for carrying out various chemical reactions. These discharges can be classified into several different categories based upon their generation mechanisms, pressure ranges, and electrode configuration. 


\section{Types of Discharges}

Radio frequency (RF) discharges are generally low-pressure reactions that do not require electrodes within the reaction space. The discharges are generated using an external induction coil. This particular process eliminates the contamination of the electrode by active species within the reactor. RF discharges operate at very high frequencies. These high frequencies produce a homogeneous reaction zone at the center of the vessel. The electrons and ions are "trapped" in the central portion of the reactor because as they accelerate in the direction of the oppositely charged portion of the field, the field changes and causes these species to change their direction.

A microwave discharge is another type of high frequency discharge. It uses a microwave radiation source to induce a discharge that must be guided into the reaction vessel by using a waveguide or waveguide resonator. The reaction volume within the discharge region decreases in size as the frequency increases. Therefore, a maximum frequency exists where the reaction volume is too small for practical applications.

A glow discharge occurs when a DC or low frequency $A C$ potential is applied across two metal electrodes sealed in opposite ends of a glass tube at low pressures. The potential required for breakdown is relatively low due to the low pressure across the electrodes. Once breakdown occurs, a visible glow appears in the region between the electrodes. The glow, however, is not uniform when a DC potential is used. The actual distribution of light in the tube is significant. Adjacent to the cathode is a very thin dark layer called the Aston Dark Space (Aston DS). 
This dark space separates the cathode from a thin cathode glow (CG). The glow is bounded on the other side by a much darker region named the Crookes or Hittorf Dark Space (CDS). For a given current, the CDS increases in width as the gas pressure decreases. Following the CDS is another luminous region called the negative glow (NG). The NG is bounded on the opposite side by an almost completely dark region named the Faraday Dark Space (FDS). Following the FDS is a long luminous region extending nearly all the way to anode referred to as the uniform positive column (PC). The anode end of the $P C$ is sometimes brighter and is called the anode glow (AG). The AG is separated from the anode by another very thin dark space called the anode dark space (ADS). These various regions are significant because the products from a reaction tend to form in zones. This spatial distribution of product selectivities can be beneficial in some processes. However, due to the low pressure and the resulting low mass flowrate in the reactor vessel, the glow discharge tubes are generally used for applications like fluorescent and neon lighting.

The corona discharge is a breakdown that occurs at moderate gas pressures between two electrodes; one of which is a point electrode while the other is a plate electrode. The nature of the discharge depends on whether the point electrode is the positive electrode or the negative electrode. The corona discharge is associated with the formation of streamers at voltages below the sparking potential. These streamers do not lead to the development of sparks and are used for the study of streamer processes. Since one of the electrodes is a point electrode, the reaction volume for the corona discharge is small. Therefore, this discharge is not useful for 
the production of large-scale materials.

The dielectric-barrier discharge, also commonly referred to as a silent electric discharge, occurs in the gas gap between a pair of electrodes that have the same geometry, that is, between two flat-plate electrodes or in the annulus between two concentric cylindrical electrodes. A dielectric barrier that is commonly made of glass covers at least one of the electrodes. This dielectric allows the system to be used at pressures up to an atmosphere. High voltage alternating current is often used to provide the electric potential across the electrodes. The resulting breakdown between the electrodes exists as a large number of micro-discharges that are uniformly spread over the reaction volume and the entire electrode surface. As ionization occurs within the gas gap, ions migrate toward the electrode with the opposite charge and collect at the surface of the dielectric. After a period of several nanoseconds, the magnitude of the charge that has accumulated at the surface of the dielectric is enough to counterbalance the potential applied across the opposite electrode. This effectively reduces the potential across the gas gap to a level that interrupts the continuous flow of electrons through the micro-discharges.

The dielectric serves two main functions during the discharge. The first is to promote an even distribution of micro-discharges across the entire reaction volume. This increases the contact time between gas molecules and high-energy electrons and therefore promotes the conversion of the gaseous species. The second function is to control the amount of charge transferred across each micro-discharge. This helps prevent the formation of arcs across the gas gap. Arcing is the rapid transfer of high-energy electrons across the gas gap in a very small volume. These 
energetic electrons are less likely to come into contact with the reactant gas, thereby making the reaction less efficient.

\section{Silent Electric Discharge Reactors (SEDR)}

Presently, the only commercial application for a silent electric discharge reactor is the generation of ozone from oxygen. Recent studies with silent electric discharge reactors involved the destruction of various toxic species like $\mathrm{NO}_{x}, \mathrm{H}_{2} \mathrm{~S}$, and volatile organic compounds in the presence of oxygen. Although no new commercially successful uses for SEDRs have been developed from this research, it shows that interest in the potential commercial applications for SEDRs is high.

The most recent work on the use of a silent electric discharge reactor for methane conversion has been conducted at The University of Oklahoma in Norman, OK. The work of Mallinson et al in 1987 showed that the reaction of methane and oxygen in a SEDR resulted in the formation of methanol and other organic compounds. The work of Bhatnager et al in 1993 expanded upon Mallinson's work and resulted in extensive data on methane conversion and product selectivity in an SEDR. 


\section{Work to be Done}

\section{Problems}

The recently completed work using a silent electric discharge reactor for the partial oxidation of methane has been very promising. However, several problems must be solved before the use of SEDRs for the conversion of methane can be economically feasible and commercially acceptable. These problems include the high selectivities of species such as carbon monoxide, carbon dioxide, and water. These products have little or no commercial value. Another problem is the relatively poor conversion of methane even at high electric potentials. In the instances where the selectivities of the desired products were at a maximum, the conversion of methane was less than $10 \%$ per pass. Another major problem is the lack of information on the effects of various physical parameters such as gas gap distance, dielectric thickness and cooling rate on the equilibrium conversion of methane and the selectivities of the desired products. This problem is due to the fact the SEDR used for these experiments was a cylindrical SEDR. With this type of reactor, the physical specifications like gap distance and dielectric thickness are fixed. Therefore, they cannot be manipulated. The last major problem is the lack of data concerning the consumption of power by the SEDR. The most significant costs associated with using a silent electric discharge reactor is the cost of the electrical utility needed to provide the necessary potential difference across the reactors electrodes for the silent electric discharge to occur. This will be the most important factor in determining whether or not the SEDR will ever be used as a commercial 
process for the conversion of methane.

\section{Potential Solutions}

The purpose of this particular research is to try and solve these problems. A systematic approach will be used to find potential solutions to these difficult problems in a new and effective way. In order to solve the first problem, it has been proposed that the oxygen be eliminated entirely from the feed stream. The advantage of this change is that the elimination of oxygen from the feed stream means that no oxygenated species will form. The only products that will form are higher hydrocarbons and hydrogen, all of which have industrial value or significant heating value. If any of the products from the reaction are deemed to be not valuable enough for further processing or transport, they can be sent to an electric generator and burned and the electricity can be used to continue the reaction in the SEDR. The main disadvantage of the removal of oxygen is the fact that oxygen has long been known to aid the activation and conversion of methane. By removing the oxygen, one of the main routes for methane conversion to more valuable products is terminated. This falls into line with the second problem - the lack of high methane conversion. In order for this change to be effective, it is necessary to find more efficient methods of activating the methane molecule. This may involve adding a chemically inert species like helium to the feed stream to enhance the activation of methane or adding a layer of a catalyst to the dielectric to aid the conversion of the various ionized or activated hydrocarbon species during the reaction.

The third problem can only be solved by changing the silent electric discharge 
reactor from a fixed cylindrical geometry to that of a flat plate geometry. In order to do this, a new reactor system had to be built that would allow the dielectric and any spacers used to maintain an even gas gap to be removed and replaced. A diagram of the new reactor can be seen in Appendix B. This reactor was initially designed to have a similar configuration to that of the original cylindrical reactor. That is, it would have a similar gas gap width, dielectric thickness, reaction area, and reaction volume as the cylindrical reactor. This was done so that results from the new reactor could be directly compared to those from the old reactor for the same conditions. Afterwards, the dielectric and/or the spacers would be replaced to test other conditions.

The rate of cooling from the water jacket can be determined by using a recycle water bath with temperature control. By measuring the temperature and flowrate of the cooling water at the inlet and the outlet of the water jacket, one can determine the amount of heat removed from the reactor at steady state. After measuring the temperature and flowrate of the inlet and outlet gas streams, the heat transfer can be calculated and its effect on methane conversion and product selectivity . 